\title{
Review article: \\ Investigation of gene markers and pathways in macrophages responsible for the Th1/Th17 inflammatory condition of Periodontal infection that can be candidates in stem cell therapy of the disease. Chikoo Cherian ${ }^{1}$, Jannet Vennila James ${ }^{2}$
}

\begin{abstract}
:
Periodontitis is defined as a chronic gum disease with characteristic inflammation attributed byTh1/Th17 showing excessive immune responses and Tregs dysregulation occurring in the macrophages. Several genes and associated pathways have been identified which influence this condition. By deploying bioinformatics approaches and tools, the authors of this paper validated the presence of the responsible genes and identified its pathways. The current study focuses on using these genes as molecular markers and inhibiting their pathways in addition to conditioning the conversion of Th17 cells into Treg cells by introducing stem cell therapy. This study specifically used Wharton jelly or Umbilical cord stem cells therapy in the control and treatment of Periodontitis.
\end{abstract}

Keywords: Periodontitis; Treg; stem cells; molecular markers; pathways; macrophages

Bangladesh Journal of Medical Science Vol. 19 No. 04 October'20. Page : 594-597 DOI: https://doi.org/10.3329/bjms.v19i4.46611

\section{Introduction}

Periodontitis is an infectious disease that has been reported to affect both the developed and developing countries. The infection manifests itself to about 20 $50 \%$ of the global human population. Periodontitis is characterized by periodontal ligament loss accompanied by the destruction of the surrounding alveolar bone ${ }^{1}$. The major risk factors that contribute to this disease are smoking ${ }^{2,3,4,5}$, hormonal changes especially in females ${ }^{6}$, poor oral hygiene ${ }^{7}$, incidence of diabetes mellitus ${ }^{8,9,10}$, certain medications ${ }^{11}$, stress $^{12}$, age ${ }^{13}$ and other hereditary related factors ${ }^{14}$. Porphyromonas gingivalis has been identified as the primary pathogen that causes this infection. This bacterium is a Gram negative oral anaerobe which contributes to the pathogenesis of the condition Periodontitis ${ }^{15}$.

Molecular expression based studies that employed microarray technology permits a genome-scale evaluation of the gene function. This analysis helps resolve the research gap in identifying specific gene expression patterns that link metabolic characteristics which contribute to the progression of the disease ${ }^{16}$. Network based approaches have been directed currently for creating a better understanding of the molecular mechanism of the disease and the associated pathways ${ }^{17}$.

Derived from the hematopoietic cell lineage, osteoclasts are differentiated from monocytes. Monocytes originate from the bone marrow into the blood and migrate to various different tissues and to further differentiate into tissue specific macrophages ${ }^{18}$. Periodontal inflammation is caused as a result of the hyperactivity of the Th1, Th17 and the dysregulation of Treg cells ${ }^{19,20}$. Thus the network study, pathway identification and analysis of the genes involved in the inflammatory pathways of the macrophages can serve as target genes that enable functions in the regulation of Treg cells and control the Th17/Treg homeostasis imbalance.

Stem cell therapy is an emerging arena applied for the treatment of autoimmune diseases. Stem cell therapy is the use of stem cells in the for the treatment and prevention of a disease or condition ${ }^{29}$. Among the many sources of stem cells, the human umbilical cord matrix i.e. Wharton's jelly has recently become

1. Chikoo Cherian, Department of Biosciences and Technology, Karunya Institute of Science and Technology

2. Jannet Vennila James, Department of Biotechnology, Karunya Institute of Technology and Sceinces

Correspondence to: Chikoo Cherian, Department of Biosciences and Technology, Karunya Institute of Science and Technology Email addresses: chikoozcherry@gmail.com (Chikoo Cherian), jannet_r@karunya. edu (JannetVennila James) 
a preferential source of stem cells. This has gained popularity because of its rapid availability with a large donor pool, non-invasive and painless collection method. The collection of this type of stem cell possesses no risk for the donor, no ethical constraints, is hypo immunogenic and non-tumorigenic. These cells have high in-vitro expandable rates and possess multi-potent differentiation potential, which identifies them as important sources for the isolation and banking of stem cells $\mathrm{s}^{21-23}$. Human Wharton's Jelly-derived Mesenchymal Stem Cells (hWJ-MSCs) display immunosuppressive properties and may thus be able to play an important role in autoimmune disorders ${ }^{24}$. WJSCs are plastic-adherent when maintained in standard culture conditions. They express CD105, CD73, and CD90in addition to recently recognized markers such as CD44, CD146, and CD166. However, they do not express CD3, CD45, CD34, CD14 or CD11b, CD45, CD144, CD79 $\alpha$ or CD19, vascular endothelial growth factor (VEGF)-R1, VEGF-R2, and HLA-DR surface molecules $^{30,31}$. Some UCB-derived cell populations show inherent 'immune-privileged' properties as they exhibit class I HLA antigens, and class II HLA antigens are observed only in response to INF- $\gamma^{32}$. These features fulfil the stipulated minimum criteria of 'plastic adherence', 'immunological profile', and 'differentiation' which were mentioned in the paper of the International Society for Cellular Therapy ${ }^{30}$. MSCs from within WJSCs are a relatively younger cells compared to most other MSCs. Regulatory T-cells (Treg) are crucial in preventing the development and progression of many autoimmune diseases development. Interleukins typically induces the proliferation of Treg cell populations and reduces the activity of Th17 and Th1 cells, which tend to play a primary role in the initiation of inflammation in autoimmune diseases. Amari et al. revealed that Wharton jelly stem cells serve as a good source of particular interleukins that enhance the reduction function associated with inflammation thus decrease the implications of the autoimmune diseases ${ }^{24}$.

\section{Methods}

The dataset (GSE24897) that was obtained from GEO (Gene Expression Omnibus) (www.ncbi. nlm.nih.gov/geo) is the expression data procured from human macrophages which were treated with Porphyromonas gingivalis bacteria.

Gene Spring is a powerful, accessible statistical tool that is used for intuitive data analysis and visualization. Gene Spring typically offers an interactive domain that promotes investigations enable comprehension of the obtained genetic data within its biological context. GeneSpring allows quick and reliable identification targets of interest that are statistically and biologically meaningful. Gene Spring is an expanding suite of integrated software applications useful for systems-level exploration, handling genomic data in single unified application models. The different stem involves normalization- intends to alter microarray data for impacts which emerge from variations in the innovation rather than from biological contrasts between the samples or printed probes. Gene Spring consists of different types of normalization algorithms which include RMA algorithm, Percentile shift, and Quantile. For Affymetrix and Agilent data sets, the GeneSpring uses RMA algorithm and Percentile shift respectively. It considers only Perfect Matches and hence uses positive signal intensities for probe level normalizations. It reduces the noise by not considering mismatches. The next step includes the percentile shift which involves the raw signal values that are transformed to $\log$ base, $\log$ transformed values which are arranged in ascending order and required percentile (75th Percentile) is then computed. To obtain the normalized intensity value the 75 th percentile value is then subtracted from corresponding log signal values ${ }^{25}$.

The top 20 up regulated genes that were determined by the GeneSpring analysis were used as an precursor in the GIANT database in order to visualize the genegene interaction network with systematic analysis and observation of the pathway that contributes to specific gene expressions. GIANT leverages a tissues specific gold standard to automatically up-weight datasets that are relevant to the specific tissue procured from a large data compendium of diverse tissues and celltypes. The resulting functional networks accurately capture various tissue-specific functional interactions that occur in the macrophages ${ }^{26}$.

\section{Results and discussion}

The top twenty up regulated genes with the highest fold change that was generated with the help of the GeneSpring analysis have been included in the following table (Table 1). Certain inflammatory genes that are present in multiple pathways in the macrophages showed high fold change on periodontal infection by the organism Porphyromonas gingivalis.

The next strategy in the current analysis was involved with systematic observation of the gene- 
gene interaction among the identified genes in addition to the interactions with corresponding neighbouring genes. The information pertaining to this was entered in the GIANT database and their respective interactions are elaborated in Figure 1. The gene-gene interaction and their network weights are mentioned in Supplementary 3.

Th17 cell homeostasis represents the relationship with Tregs, where its imbalance leads to the development of Periodontitis. Stem cells possess the property of therapeutic agents that were associated with an increase in CD4+FoxP3+ Treg cells in addition to inhibition of Th17 cell formation and systematic inhibition of osteoclast differentiation ${ }^{27}$. They reciprocally modulate FoxP3 and ROR $\gamma$ t expressions which eventually facilitate the conversion of Th17 into Treg cells. CD4+IL17+FoxP3+ROR $\gamma \mathrm{t}-\mathrm{T}$-cells indeed represents an intermediate phenotype of Th17 cells that are modified into Treg cells ${ }^{28}$. The genes that were expressed in the macrophages (Table 1) serve as molecular markers of Periodontitis. Identification and understanding of these genes and their gene expression can enable the control of progression and enhance the treatment regimes for eradicating Periodontitis.

\section{Conclusion}

The data presented in the current study suggests that the up regulated genes that were identified through microarray analysis (GeneSpring) form the basis of identifiable molecular targets that can be treated by stem cell therapy. This research particularly indicates the application of Wharton Jelly or Umbilical cord stem cell therapy. Furthermore this study suggests that gene clusters that form pathways (Table 2) which contribute to Periodontal inflammation can be methodically targeted and inhibited. This study paved way for a novel approach in the treatment of Periodontitis with the help of Stem cell therapy techniques.

\section{References:}

1. De pablo P, Chapple IL, Buckley CD, Dietrich T. Periodontitis in systemic rheumatic diseases. Nat Rev Rheumatol. 2009;5(4):218-24.

2. Sanz M, D'Aiuto F, Deanfield J, Fernandez-Avilés F. European workshop in periodontal health and cardiovascular disease-scientific evidence on the association between periodontal and cardiovascular diseases: A review of the literature. Eur Heart J Suppl. 2010;12(Suppl B):B3-12.

3. Polk DE, Wang X, Feingold E, et al. Effects of smoking and genotype on the PSR index of periodontal disease in adults aged 18-49. Int $J$ Environ Res Public Health.
2012;9(8):2839-50.

4. Albandar JM, Streckfus CF, Adesanya MR, Winn DM. Cigar, pipe, and cigarette smoking as risk factors for periodontal disease and tooth loss. J Periodontol. 2000;71(12):1874-81.

5. Reners M, Brecx M. Stress and periodontal disease. Int J Dent Hyg. 2007;5:199- 204.

6. Güncü GN, Tözüm TF, Caglayan F. Effects of endogenous sex hormones on the periodontium - Review of literature. Aust Dent J. 2005;50:138-45.

7. de Oliveira C, Watt R, Hamer M. Toothbrushing, inflammation, and risk of cardiovascular disease: Results from Scottish Health Survey. BMJ. 2010;340:c2451. 
8. Casanova L, Hughes FJ, Preshaw PM. Diabetes and periodontal disease: A two-way relationship. Br Dent $J$. 2014;217:433-7.

9. Chávarry NG, Vettore MV, Sansone C, Sheiham A. The relationship between diabetes mellitus and destructive periodontal disease: A meta-analysis. Oral Health Prev Dent. 2009;7:107-27.

10. Preshaw PM, Bissett SM. Periodontitis: Oral complication of diabetes. Endocrinol Metab Clin North Am. 2013;42:849-67.

11. Güncü GN, Tözüm TF, Caglayan F. Effects of endogenous sex hormones on the periodontium - Review of literature. Aust Dent J. 2005;50:138-45.

12. Reners M, Brecx M. Stress and periodontal disease. Int J Dent Hyg. 2007;5:199- 204.

13. Grodstein F, Colditz GA, Stampfer MJ. Post-menopausal hormone use and tooth loss: A prospective study. $J \mathrm{Am}$ Dent Assoc. 1996;127:370-7.

14. Casanova L, Hughes FJ, Preshaw PM. Diabetes and periodontal disease: A two-way relationship. Br Dent J. 2014;217:433-7.

15. Jaroslav Mysak, Stepan Podzimek, PavlaSommerova, et al., "Porphyromonasgingivalis: Major Periodontopathic Pathogen Overview," Journal of Immunology Research, vol. 2014, Article ID 476068, 8 pages, 2014.

16. Barabási. A. L., Gulbahce. N. and Loscalzo. J. (2011) "Network medicine: a network-based approach to human disease", Nature Reviews Genetics 12, 56- 68.

17. Zanzoni. A., Soler-López. M. and Aloy. P. (2009) “A network medicine approach to human disease", FEBS letters 583, 1759-1765.

18. Geissmann F, Manz MG, Jung S, Sieweke MH, Merad M, Ley K. Development of monocytes, macrophages, and dendritic cells. Science 2010; 327: 656-661.

19. Rodan GA, Martin TJ. Therapeutic approaches to bone diseases. Science. 2000;289:1508-14.

20. Walsh MC, Kim N, Kadono Y, Rho J, Lee SY, Lorenzo J, et al. Osteoimmunology: interplay between the immune system and bone metabolism. Annu Rev Immunol. 2006;24:33-63

21. Batsali, A.K.; Kastrinaki, M.C.; Papadaki, H.A.; Pontikoglou, C. Mesenchymal stem cells derived from Wharton's Jelly of the umbilical cord: Biological properties and emerging clinical applications. Curr. Stem Cell Res. Ther. 2013, 8, 144-155.

22. Richardson, S.M.; Kalamegam, G.; Pushparaj, P.N.; Matta, C.; Memic, A.; Khademhosseini, A.; Mobasheri, R.; Poletti, F.L.; Hoyland, J.A.; Mobasheri, A. Mesenchymal stem cells in regenerative medicine: Focus on articularcartilage and intervertebral disc regeneration.
Methods 2016, 99, 69-80.

23. Sabapathy, V.; Sundaram, B.; Mankuzhy, P.; Kumar, S. Human Wharton's Jelly Mesenchymal Stem Cells plasticity augments scar-free skin wound healing with hair growth. PLoS ONE 2014, 9, e93726.

24. Amari, A.; Ebtekar, M.; Moazzeni, S.M.; Soleimani, M.; Mohammadi-Amirabad, L.; Tahoori, M.T.; Massumi, M. In vitro generation of IL-35-expressing Human Wharton's Jelly-derived Mesenchymal Stem Cells using lentiviral vector. Iran. J. Allergy Asthma Immunol. 2015, 14, 416-426.

25. Chu. L., Scharf. E. and Kondo. T. (2001) "GeneSpring TM: Tools for Analyzing Microarray Expression Data", Genome Informatics 12, 227-229.

26. Greene $\mathrm{CS}^{*}$, Krishnan $\mathrm{A}^{*}$, Wong $\mathrm{AK}^{*}$, Ricciotti $\mathrm{E}$, Zelaya RA, Himmelstein DS,Zhang R, Hartmann BM, Zaslavsky E, Sealfon SC, Chasman DI, FitzGerald GA,Dolinski K, Grosser T, Troyanskaya OG. (2015). Understanding multicellularfunction and disease with human tissue-specific networks.Nature Genetics.

27. Park, M.J.; Park, H.; Cho, M.L.; Oh, H.J.; Cho, Y.G.; Min, S.Y.; Chung, B.H.; Lee, J.W.; Kim, H.Y.; Cho, S.G. Transforming growth factor beta-transduced mesenchymal stem cells ameliorate experimental autoimmune arthritis through reciprocal regulation of Treg/Thl7 cells and osteoclastogenesis. Arthritis Rheum. 2011, 63, 1668-1680.

28. Prasanna, S.J.; Gopalakrishnan, D.; Shankar, S.R.; Vasandan, A.B. Proinflammatory cytokines, IFNgamma and TNFalpha, influence immune properties of human bone marrow and Wharton jelly mesenchymal stem cells differentially. PLoS ONE 2010, 5, e9016.

29. Mahla RS (2016). "Stem cells application in regenerative medicine and disease threpeutics". International Journal of Cell Biology. 2016 (7): 1-24.

30. Damien, P.; Allan, D.S. Regenerative therapy and immune modulation using Umbilical Cord BloodDerived Cells. Biol. Blood Marrow Transplant. 2015, 21, 1545-1554.

31. Dominici, M.; Le Blanc, K.; Mueller, I.; SlaperCortenbach, I.; Marini, F.; Krause, D.; Deans, R.; Keating, A.; Prockop, D.; Horwitz, E. Minimal criteria for defining multipotent mesenchymal stromal cells. The International Society for Cellular Therapy position statement. Cytotherapy 2006, 8, 315-317.

32. Chamberlain, G.; Fox, J.; Ashton, B.; Middleton, J. Concise review: Mesenchymal stem cells: Their phenotype, differentiation capacity, immunological features, and potential for homing. Stem Cells 2007, 25, 2739-2749. 